\title{
EL EFECTO DE LA ENTROPÍA EN LOS SOFTWARES MULTIMEDIALES EDUCATIVOS
}

Luis Rodolfo Lara

Universidad Nacional de Catamarca, Argentina

\section{INTRODUCCIÓN}

Ante la presencia de innumerables estrategias y métodos que se utilizan para el desarrollo de softwares multimediales educativos, en donde el diseño no se encuadra bajo un claro sustento teórico o están enmarcados en teorías prestadas que corresponden a la modalidad presencial o en determinadas reglas aisladas de diseño, se hace necesario realizar un análisis de estos materiales con una nueva perspectiva en lo que respecta a su principio de desarrollo, desde un horizonte sistémico e interdisciplinario.

El carácter interdisciplinario de este trabajo se basa, justamente en la estructura compleja que presentan estos elementos interactivos, como lo expresa Morin (1999) "los desarrollos disciplinarios de las ciencias no sólo aportaron las ventajas de la división del trabajo, también aportaron los inconvenientes de la superespecialización, del enclaustramiento y de la fragmentación del saber. No produjeron solamente conocimiento y elucidación, también produjeron ignorancia y ceguera" (p. 15). Por consiguiente, incorporando principios y conceptos teóricos provenientes de diversas disciplinas y teorías como la física, la química, los sistemas y procesos de enseñanza-aprendizaje entre otras, se busca abrir un nuevo enfoque para el diseño de materiales educativos interactivos partiendo de un modelo complejo.

El pensamiento complejo comenzó a trascender lo interdisciplinario, sin embargo, desde el punto de vista del diseño de programas multimediales educativos, no existe un panorama claro de su aplicación, este artículo pretende iluminar esa zona todavía en penumbras.

\section{UN ENFOQUE SISTÉMICO DE LOS SOFTWARES MULTIMEDIALES EDUCATIVOS}

La teoría de los sistemas provee de un terreno fértil para llevar a cabo el estudio del diseño de los materiales multimediales educativos; Grün (1999) menciona una definición de sistema de Francois como una entidad autónoma dotada de una cierta permanencia y constituida por elementos interrelacionados que forman subsistemas estructurales y funcionales, que se transforma dentro de ciertos límites de estabilidad, gracias a regulaciones internas que le permiten adaptarse a las variaciones de su entorno específico (por ejemplo, un hombre, un automóvil, una ameba). Una de las virtudes esenciales de la Teoría General de los Sistemas (T.G.S.) planteada por Bertalanffy es la de tratar a los sistemas, sin prescindir de sus relaciones con su entorno manteniendo, además, las conexiones internas y externas de sus elementos. Todo lo cual no puede ser separado sin destruir la esencia del sistema, es decir, su unidad. Pues una de las ideas básicas en T.G.S. es que el todo es más ( $\mathrm{y}$ es otra cosa) que la suma de sus partes porque las características constitutivas de ese todo no son explicables a partir de las características de las partes aisladas. 
Desde un punto de vista sistémico un software interactivo educativo tendría las siguientes características:

- Tiene una perspectiva holística: el todo es superior o diferente a las partes constitutivas

- Es recursivo y sinérgico: se la puede considerar sistema ya que se muestra independiente y coherente.

- Es un sistema abierto, es decir, un sistema real, aquel que interactúa con el entorno, pero a la vez se diferencia de él, por lo tanto:

- sufre transformaciones estructurales en igual medida que el contexto sufre transformaciones (es homeostático) y

- es permeable a los estímulos externos.

- Es un sistema realimentado, es decir, de acuerdo a las señales de entrada, el sistema por sí solo se reorienta o replantea continuamente su situación (principio de la cibernética). De este modo, el sistema tiene una realimentación positiva, es decir, representa la variación de un elemento que se propaga por el lazo de realimentación y que refuerza su valor inicial haciéndola mayor (amplificándola). En la medida que implican la instauración de nuevas estructuras, el sistema sufre procesos irreversibles y amplificados.

Partiendo de esta perspectiva sistémica y considerando el software multimedia como un Sistema Interactivo Multimedia (SIM) educativo, nos permitirá realizar un estudio más detallado de su interacción (estímulos y realimentaciones) con el entorno. Mediante un análisis entrópico será posible conocer la orientación que lleva a cabo la evolución de estos materiales al interactuar como sistema abierto, ya que en ese entorno se encuentra entre otros elementos la vinculación y la información que proporciona el usuario, de esta manera, un SIM se comportaría en forma adecuada y adaptada al perfil del usuario.

Es así, que en la actualidad, las aplicaciones multimedias educativas, en su afán de formar, sólo lo puede conseguir con un "usuario estándar", del cual los programadores y diseñadores de la aplicación tomaron como modelo con argumentos dudosos y discutibles sobre lo que significa realmente el término "usuario estándar", sin considerar que cada individuo construye su conocimiento de diversas formas y proviene de conocimientos y acontecimientos previos diferentes. Como expresa Gardner (2003) se enseñan y evalúan las materias de la misma manera, a todos los estudiantes por igual, porque parece justo poder tratar a todos los estudiantes como si fueran semejantes; se apoyan en el supuesto equivocado de que todas las personas tienen el mismo tipo de mente.

Por esta razón, un SIM educativo debe ser capaz de:

- presentar información textual, sonora y visual de modo coordinada e integrada,

- mostrar un desarrollo de contenidos y actividades en forma ramificada (tanto transversal como longitudinal),

- adaptar los contenidos y actividades a las características del alumno (tanto cognitiva como de los conocimientos previos que presenta),

- ajustarse a las características del hardware informático que se disponga, 
- comunicar y compartir información con otros alumnos (contribuya al aprendizaje colaborativo),

- poder acceder a materiales de estudio de otras fuentes (acceso a la información distribuida),

- $\quad$ exhibir contextos (modelos, aplicaciones, contenidos y actividades) adaptados por el usuario.

Desde esta perspectiva, se debe tener en cuenta que las aplicaciones informáticas que existen en la actualidad tienden a ser sistemas abiertos muy limitados, en el sentido de que los estímulos provenientes del exterior y sus realimentaciones no influyen en forma determinante en cuanto a la esencia del funcionamiento y al desarrollo del sistema.

La perspectiva sistémica y su relación con el entorno nos hace introducir otro concepto necesario para el entendimiento del desarrollo de materiales interactivos multimediales: la entropía.

\section{LA ENTROPÍA EN LOS SISTEMAS INTERACTIVOS}

El concepto de entropía, proveniente de la segunda ley de la termodinámica, tiene diversas interpretaciones que mas allá de ser diferentes son complementarias, pasa de ser una medida de la pérdida de calor, de energía (Clausius), a una medida de aleatoriedad o desorden (Boltzman) y a la vez está relacionada con la cantidad de información que se puede transmitir por un canal (Shannon y Weaver).

El análisis de la entropía puede realizarse considerando los dos tipos de sistemas que pueden presentarse.

\subsection{Entropía en los sistemas cerrados}

Los sistemas cerrados (sistemas en los cuales las transformaciones implicadas quedan todas incluidas en ellos) tienen la tendencia al crecimiento de la entropía, es decir, la máxima probabilidad de los sistemas es su progresiva desorganización y, finalmente, su homogeneización con el ambiente y se dirigen irremediablemente al desorden total (muerte térmica). La segunda ley de la termodinámica afirma que la entropía de un sistema cerrado nunca puede decrecer. Por lo tanto, cuando un sistema de este tipo alcanza una configuración de máxima entropía, ya no puede experimentar cambios: ha alcanzado el equilibrio.

Desde esta perspectiva surgen tres puntos de vista para estudiar la entropía.

\section{1) La entropía y el calor}

Clausius formuló la segunda ley enunciando que no puede pasar el calor de un cuerpo frío a otro caliente sin intervención de ningún agente externo. Por lo tanto, a este proceso se lo considera no espontáneo.

La segunda ley o principio impone una condición adicional a los procesos termodinámicos, no basta con que se conserve la energía y cumplan así el primer principio de la termodinámica. Una máquina que realizara trabajo violando el segundo principio se denomina "móvil perpetuo de segunda especie", ya que podría obtener energía continuamente de un entorno frío para realizar trabajo en un entorno caliente sin coste alguno. A veces, el segundo principio se formula como una afirmación que descarta la existencia de un móvil perpetuo de segunda especie.

Es más oportuno trabajar con el concepto de entropía en función de incrementos en un sistema en forma de diferenciales, de esta forma se presenta la definición de entropía: 


$$
d S=\frac{q}{T}
$$

Donde:

$d S$ = variación de la entropía

$q$ = calor producido en forma reversible

$T=$ temperatura del cuerpo

\section{2) La entropía y la probabilidad}

Castellan (1987) plantea el siguiente ejemplo en donde nos elucida el concepto de entropía desde una perspectiva probabilística, considérese un salón grande sellado y evacuado, en un rincón del salón hay una pequeña caja que contiene un gas, se retiran los lados de la caja y las moléculas del gas quedan en libertad para salir a la habitación. Al cabo de un tiempo, se observa que el gas se ha distribuido uniformemente en ésta. Imaginemos ahora que en un momento posterior cada componente de la velocidad de cada molécula se invierte. Entonces, las moléculas sólo invertirán su movimiento original, y después de un periodo de tiempo el gas se juntará en el rincón de la habitación donde se encontraba originalmente sellada la caja. Lo extraño es que no hay razón para suponer que el movimiento determinado que condujo a la dispersión del gas en la habitación sea más probable que el mismo movimiento invertido, el que llevo al gas a juntarse en una esquina de la habitación. Si esto es así ¿por qué nunca dbservamos que el aire de una habitación se junte en una porción determinada de la habitación?

El hecho de que nunca observemos algunos movimientos del sistema, que son inherentemente tan probables como los que observamos, se conoce como paradoja de Boltzmann. Esta paradoja se resuelve de la siguiente manera, es cierto que un movimiento de las moléculas exactamente determinado es tan probable como otro movimiento también exactamente determinado. Pero también es cierto que de todos los movimientos posibles exactamente determinado de un grupo de moléculas, el número total de los que llevan a una ocupación uniforme del espacio disponible es enormemente mayor que el número de movimientos que conducen a la ocupación de solo una pequeña parte del espacio disponible. Por lo tanto, aunque todos los movimientos individuales de un sistema tienen la misma probabilidad, la probabilidad de encontrar un espacio uniformemente es proporcional al número total de movimientos que se producen en esta situación. En consecuencia la posibilidad de observar una oupación uniforme es muchísima mayor comparada con la probabilidad de cualquier otra situación. En este caso particular se llegó al final a un estado de equilibrio, en donde todas las moléculas están distribuidas en forma desordenada en el espacio, lo que significa que se llegó a una entropía máxima.

De esta manera, se llega a la reformulación del concepto de entropía relacionado con la probabilidad definida por Boltzmann como un número de arreglos posibles de las partículas que componen el sistema y que están en consonancia con el estado del sistema:

$$
S=k \cdot \ln \Omega \quad(\mathrm{I})
$$

Donde:

$\mathrm{k}=$ constante de Boltzmann 
$\Omega=\frac{N !}{N_{a} !\left(N-N_{a}\right) !}$ es el número de configuraciones posibles del sistema en consonancia con el estado específico del mismo

$N=$ número de celdas

$N_{a}=$ número de partículas $\mathrm{A}$

\section{3) Entropía y la cantidad de información}

Por otra parte, Shannon y Weaver plantearon el concepto de cantidad de información I como el número de símbolos posibles que representan el mensaje expresado en bits y queda enunciado de la siguiente manera:

$$
I=\log _{2} \frac{1}{p(A)}
$$

Donde:

$A$ es el suceso

$p(A)$ es la probabilidad del mensaje o del suceso y es un número entre 0 (un evento improbable) y 1 (un evento posible).

Puede deducirse de la fórmula que cuanto más improbable (próximo a cero) sea un suceso susceptible de ser transmitido como un mensaje, tanto mayor es la cantidad de información que se podrá proporcionar por su ocurrencia.

A la entropía $H$, Shannon la definió como la cantidad de información promedio de estos mensajes, definida de la siguiente forma:

$$
H=\log _{2} N \quad \text { (II) }
$$

Donde $N=$ número de mensajes posibles en el conjunto

Resulta indudable que tanto la fórmula (I) como la (II) son expresiones equivalentes, diferenciándose solo en constantes.

\subsection{Entropía en los sistemas abiertos}

Ahora bien, en el mundo real, los sistemas son abiertos (donde se intercambia energía, materia o información con el entorno) y el estado más probable de esos sistemas es el caos, el desorden y la desorganización.

Desde ahora en adelante, refiriéndonos a sistemas informáticos, se considerará que lo que se transmite o recibe el sistema son flujos de información, cabe aclarar que la información tiene un comportamiento distinto al de la energía, pues su comunicación no elimina la información del emisor o fuente, cosa que sucede cuando se transfiere energía como el calor. La información es la más importante corriente negentrópica (entropía negativa) de que disponen los sistemas complejos. 
La realidad muestra como los organismos vivos como las estructuras artificiales creadas por el hombre presentan una tendencia a una mayor heterogeneidad y a unos niveles crecientes de organización. Ello es debido a que el incremento constante de la entropía que se produce en todo sistema es contrarestado en los sistemas abiertos por la importación de entropía negativa, gracias precisamente a esos flujos de información, energía, cambios de objetivos o estructuras hacen que la entropía del sistema incluso pueda disminuir.

Es claro que estos flujos producen perturbaciones e inestabilidades en el sistema pero la asimilación adecuada de los mismos, no su eliminación o neutralización, es la que permitirá que el sistema siga funcionando.

De este modo, como afirma Sarabia (1995) "en todo sistema abierto, deben tener en todo momento despejado sus canales de información con el entorno y asumir el conflicto que supone la aceptación de la diversidad, ya que en caso contrario, evolucionarán como los sistemas cerrados alcanzando su particular muerte térmica y marcándose como fin una degradación del fin original” (p. 56). Por lo tanto, permeabilizar la frontera entorno-sistema nos llevará a un terreno de inestabilidad, pero a la vez, si el sistema está bien diseñado, nos conducirá a un nivel más evolucionado del mismo.

Prigogine realiza una extensión del segundo principio de la termodinámica para sistemas abiertos (segundo principio generalizado de la termodinámica):

$$
d S=d_{i} S+d_{e} S
$$

Donde:

$d S=$ variación de la entropía a través del tiempo

$d_{i} S=$ variación de la producción de entropía interna del sistema debido a la irreversibilidad de los procesos que en él tiene lugar

$d_{e} S=$ variación del flujo de entropía que se produce con el intercambio con el medio o entorno

En termodinámica, "un proceso reversible es aquel que puede ejecutar en direcciones opuestas, de manera que todos los cambios que ocurran en cualquier parte del proceso directo se revierten exactamente en las etapas correspondientes del proceso inverso, y en los sistemas externos únicamente ocurren cambios infinitesimales" (Glasstone, 1972, p. 180) y $d_{i} S=0$; en cambio un proceso irreversible no se puede volver a una etapa anterior ya que éstas presentan un alto grado de inestabilidad.

De esta manera, todo proceso natural o espontáneo, es decir, aquel proceso que tiene lugar sin ninguna interferencia externa, es irreversible y su $d_{i} S>0$, ya que el sistema emite información, "disipa energía"; que es el caso de los softwares informativos como las enciclopedias multimedia, por ejemplo. En informática y específicamente refiriéndonos al hardware, esta "disipación de energía" se hace evidente en el aumento del tráfico y capacidad de almacenamiento de la información en la memoria RAM, como también en el mayor trabajo en el manejo de instrucciones que tiene que hacer el microprocesador para gestionar y ejecutar rutinas relacionadas con el flujo de información que se va manipulando. Un flujo continuo de esta naturaleza, llevaría al caos al sistema informático (por el nivel altísimo de la cantidad de información que se 
haría inmanejable) tanto por las limitaciones técnicas que presentan el hadware y los dispositivos como por el ambiente anárquico que producirá la saturación de la información almacenada.

Para que el flujo de entropía sea $d_{e} S<0$ (entropía negativa, neguentropía), el sistema no sólo importa información del ambiente para mantener el sistema organizado sino que tiene diversos medios para organizar y discriminar esa información, por lo tanto, para mantener ese estado de organización se hace necesario la existencia de elementos softwares que tengan la función de organizar, ordenar y manejar el flujo de la cantidad de información interna y la que proviene del exterior; así de acuerdo al valor que tome $\left|d_{e} S\right|$, podremos concluir si el sistema abierto:

- está en equilibrio $d S=d S_{M A X}>0$ (máximo nivel de desorden, estado de máxima probabilidad, todos los elementos del sistema tienen la misma jerarquía)

- va hacia el equilibrio $\left|d_{e} S\right|<\left|d_{i} S\right| \Rightarrow d S>0$ (alto nivel de desorden, todos los elementos tienden a tener la misma jerarquía)

- está lejos del equilibrio $d S \leq 0$, el sistema tiende a una estructura organizada de orden superior.

En este caso se puede presentar tres situaciones:

- $\left|d_{e} S\right| \cong\left|d_{i} S\right| \Rightarrow d S \approx 0$ (tiende al orden total, los elementos del sistema se encaminan a una estructura organizada)

- $\left|d_{e} S\right|=\left|d_{i} S\right| \Rightarrow d S=0 \quad$ (orden total, los elementos tienen una estructura totalmente organizada, grado estacionario)

- $\left|d_{e} S\right|>\left|d_{i} S\right| \Rightarrow d S<0$ (autoorganización, estructuras disipativas de Prigogine), en este caso se presenta en forma espontánea y al azar estructuras y tipos de ơganización. Mientras un sistema abierto sea capaz de seguir alimentándose de una entropía negativa, tenderá a estados de mayor orden y de mayor improbabilidad.

Entonces, en un sistema abierto en constante crecimiento, para evitar que el desarrollo del mismo se vuelva caótico es fundamental que el proceso de evolución del mismo se de en un alto nivel de organización $(d S \approx 0)$.

\subsection{Hacia la zona cero de la variación de la entropía}

Como afirma Atkins (1991), los cambios espontáneos siempre van acompañados de una reducción en la "calidad" del flujo de la energía, materia o información, en el sentido de que es degradada a una forma más dispersa y caótica, eso en el concepto de entropía se traduce en un aumento de la misma a un valor mucho mayor a cero $(d S>>0)$.

En el desarrollo de SIM se debe considerar que su interacción con el entorno, nos lleve a un nivel controlable de organización, por lo que se deduce que la variación total de entropía debe ser próxima a cero 
( $d S \approx 0$, zona cero entrópica), es decir la entropía permanece constante, de esta manera, la cantidad de información que adquiere el sistema se ve jerarquizada y valorada (el sistema habrá evolucionado a un estado superior de organización).

Por lo tanto, se busca considerar al software multimedia educativo como un sistema abierto con una estructura básica (con contenidos diversos) que, para que vaya desarrollándose precisará sustentarse de señales proveniente del entorno, de donde le suministrará de información adicional para neutralizar la entropía creciente del sistema, de esta forma irá expandiéndose pero en forma ordenada en el desarrollo de módulos que se generarán de acuerdo a los requerimientos que solicite el entorno (perfil del alumno + hardware) en consecuencia, sin los componentes neguentrópicos que debe contener el sistema, éste se orientaría indefectiblemente a un estado kjos del equilibrio $(d S>0)$ y caótico. Lo que se busca con la inclusión de estos elementos, es que el software vaya creciendo por la acumulación seleccionada de información, proveniente de datos del entorno (principalmente proporcionada por la interacción con el alumno); proveyendo el sistema como respuesta, tanto de actividades como de contenidos adecuadas a esas interacciones.

Ese crecimiento no será en forma indiscriminado y desordenado, ya que el software contará con elementos para poder controlar y describir los trayectos y el seguimiento de los contenidos más adecuados para el perfil del alumno que está interactuando (de esta manera, estamos organizando el sistema, mediante una variación entrópica cercana a cero).

Así se puede asimilar que los SIMs son sistemas abiertos que toman datos y parámetros del entorno, asimismo, se desarrollan diversos módulos, aumentando la entropía, pero por otro lado existen otros elementos que componen este sistema que tratan de organizarlo, por lo tanto, se configurará un sistema que esté lejos del equilibrio pero organizado, ya que no se justifica un software educativo tendiendo al desorden.

\section{PERFIL CONSTRUCTIVISTA EN EL DISEÑO DE SOFTWARES EDUCATIVOS}

Desde el punto de vista didáctico de estos materiales interactivos, lo relevante del enfoque constructivista es que el alumno tiene el control del proceso, en el momento en que el aprendiz toma decisiones que emparejan de acuerdo a su propio estado cognoscitivo y de sus propias necesidades. Squires (1999) afirma que se presenta una paradoja si aceptamos una visión constructivista del aprendizaje con respecto al diseño de los programas multimediales educativos: si al intentar diseñar ambientes de aprendizaje eficaces estamos al mismo tiempo reprimiendo los niveles de libertad para que los mismos aprendices puedan tomar decisiones sobre su propio aprendizaje.

De acuerdo a las intenciones de diseño que se tuvo a realizar un programa multimedia educativo, los usuarios con fines educativos pueden subvertir el diseño de software para satisfacer sus propias necesidades en diferentes grados, según los objetivos que se persiguen en las actividades y el grado de delegación que permite el software, es decir, a través de la manera en la que usan al software, maestros y alumnos pueden reformar las intenciones originales que tuvo el diseñador. Desde una perspectiva del diseño Squires la llama "subversión delegada". Esta delegación puede presentarse en tres niveles, dependiendo en si el software incorpora intenciones del diseño educativas explícitas, implícitas o ausentes. El uso subversivo de software en la primera categoría requerirá un esfuerzo consciente por frustrar las 
intenciones de los diseñadores. En el segundo caso, el software puede usarse subversivamente sin que el usuario esté consciente de él. En el tercer caso, por definición, una ausencia de apuntalar intenciones del diseño educativo, a menudo debido a que el software originalmente fue pensado para uso general, por lo que es "secuestrado" para los propósitos educativos, en este caso, se pone la carga en el maestro y/o aprendiz para subvertir las intenciones no-educativas del diseñador.

En cuanto a "la subversión incorporada", consiste en dar énfasis al papel activo y determinante de los alumnos en configurar sus propios ambientes de aprendizaje para resonar de acuerdo a sus propias necesidades, haciendo eco de las nociones de aprendizaje con tecnología a través del "compromiso atento" y los aprendices como diseñadores intentando representar su propio conocimiento, de esta manera se busca la significancia personal. La subversión incorporada lleva a diseños del software que son volátiles por naturaleza, respondiendo al cambio y necesidades idiosincrásicas de aprendices. Un rasgo crucial de subversión incorporada es la creación de un enlace entre la exploración y expresión proporcionando una realimentación intrínseca que representa los efectos de las acciones de los alumnos.

De esta manera, los programas deben ser volátiles con respecto a su diseño y subversivo en cuanto a su utilización. La idea de volatilidad está proporcionando de alguna manera, la idea del grado de permeabilidad del sistema abierto con respecto a la estimulación del entorno, y lo subversivo, con el nivel de maleabilidad que pueden presentar, mediante el proceso adecuado del tránsito de información tanto interna como aquella proveniente del entorno que llevará a cabo el software.

\subsection{Hacia los softwares no programables}

Los programas actuales de computación referidos a usuarios finales presentan secuencias preestablecidas de acciones, son "secuencias de instrucciones escritas en un lenguaje determinado y que vienen a representar la solución a un problema" (Sánchez Cerezo, 1991, p. 429), no obstante, para enfrentar la incertidumbre de la acción es necesario el recurso de la estrategia, que permite a partir de una decisión inicial, imaginar un cierto número de escenarios para la acción, escenarios que podrán ser modificados según las informaciones que lleguen al sistema en el curso de la acción y según los elementos aleatorios que sobrevendrán y perturbarán esa acción.

Morin (1999) expresa que la estrategia debe prevalecer sobre el programa, ya que éste al presentar secuencias determinadas de antemano en un entorno estable, al ocurrir alguna alteración en las condiciones externas, se bloquea. En cambio, la estrategia elabora un escenario de acción examinando las certezas e incertidumbres de la situación, las probabilidades y las improbabilidades, se pueden plantear estrategias utilizando secuencias cortas de programas, pero para toda aquello que se efectúe en un entorno inestable e incierto, como sucede en el proceso enseñanza-aprendizaje, debe imponerse la estrategia.

Si se considera que el proceso de enseñanza y aprendizaje es complejo y que cada individuo aprende de distinta manera, las aseveraciones de Morin desde una perspectiva general deben ser incluidas en el ámbito del diseño de los programas multimediales educativos, por lo tanto, para que el proceso pueda ser efectivo y eficaz, los mencionados programas que existen en la actualidad deben dejar de ser programados para elevarse (o descender, según el punto de vista) a otra condición, la de ser un medio que no sólo almacene y presente información sino que realmente pueda formar al individuo planteando diversas estrategias de acuerdo a señales realimentadas implicadas en el proceso de enseñanza-aprendizaje. 
Por lo tanto, respaldamos el desarrollo de un nuevo software que va mas allá de lo intangible, un software volátil, modular como el tradicional pero con un crecimiento no sólo longitudinal sino también transversal y a la medida de las necesidades del alumno, sin limitaciones y adaptable al usuario, cambiando éste el rol pasivo que siempre se le dio para transformarse en el verdadero protagonista del proceso, constituyéndose también en el diseñador de su propio software.

\subsection{El desarrollo de contenidos modulares}

Basándonos en el acercamiento en espiral para aprender de Bruner citado por van Dam (2002), donde un alumno encuentra un tema varias veces a lo largo de su educación, cada vez a un nivel creciente de sofisticación, además, en cualquier fase en que se está aprendiendo, uno debe poder combinar y equiparar módulos educativos a los niveles diferentes de sofisticación dentro de la misma área del tema general, Gimeno Sacristán y Pérez Gómez (1995) resumen los postulados del pensamiento de Piaget: el aprendizaje no es hereditario sino que es construido por el individuo a través de un proceso dinámico de desarrollo y transformación de estructuras que dialécticamente evolucionan en espiral. Este proceso de construcción genética se realiza a través de la asimilación de los conocimientos nuevos al integrarse a las anteriores estructuras; y la acomodación, reformulación y elaboración de nuevas estructuras como consecuencia del proceso de asimilación. El conocimiento es una elaboración subjetiva y no una œpia figurativa de lo real.

De esta manera, para el desarrollo de contenidos de los SIMs deberá presentarse considerando dos aspectos:

- contenidos basados en diversos modelos adaptables a cada usuario (de acuerdo a diversas características cognitivas, culturales, por ejemplo)

- grado de complejidad de los mencionados modelos según las características cognitivas, de inteligencia y de conocimientos previos que presente el alumno.

El grado de complejidad tanto de los modelos como de la sofisticación que presente cada uno de ellos presenta una gran variedad de alternativas para presentar los contenidos para cada nivel particular que requiera el alumno.

Cada uno de estos módulos estará conectado en forma bidireccional con el resto de los módulos, de esta forma, el usuario al interactuar con el software, describirá el trayecto a seguir en cualquier dirección según las necesidades y opciones que quiera el alumno.

En un sistema abierto como son los SIMs, en la práctica sólo es significativo aquel entorno o ambiente a una parte del universo con el cual el sistema mantiene intercambios de cierta importancia y de una manera más o menos frecuente, entonces, este entorno determinará el rol que tendrá el cbcente cuando interactúe entre el software y el aprendiz.

Así el entorno de nuestro sistema estará compuesto por:

a) Las características del usuario

- las capacidades cognitivas,

- las estrategias de aprendizaje que emplea, 
- los conocimientos previos que posee

b) Las características técnicas informáticas que posee la computadora (hardware y software disponible),

- microprocesador, memoria disponible, hardware en general

- $\quad$ sistema operativo donde trabajará la aplicación, recursos o software de base que son necesarios

c) Los medios alternativos (auxiliares o secundarios) que intervendrán en el proceso de enseñanzaaprendizaje (material escrito, pizarra, otros medios de comunicación, etc.)

Este entorno así definido, presenta diversas variables, que hace que la aplicación trabaje en forma personalizada.

Así, los SIMs estarán constituidos por pequeñas células que podrán interactuar una o varias de ellas (de acuerdo a diversas condiciones que presente el entorno) en el desarrollo de una aplicación multimedia, el nuevo modelo de una aplicación multimedia educativa estará formada por diversos módulos, cada uno de ellos con una identidad propia pero a la vez formando en su conjunto una entidad única multimedial que interactuará con el entorno.

Toda la aplicación multimedia estará conformada por pequeños módulos programados que solamente describirán acciones inmediatas, formando un verdadero sistema, así, el itinerario arbóreo que se recorre dependerá de los caminos previos recorridos por el usuario y por las decisiones que tome del marco hipertextual, por las características del entorno, por interacciones detectadas por la aplicación y por acciones que tomará el alumno de acuerdo a diversos eventos que se presente. Por lo tanto, el camino que llevará al alumno por diversos itinerarios no sólo estará definido por lo hipervínculos en el que estudiante por voluntad propia intentará recorrer, sino por los diversos módulos (conectados en paralelo y en serie y bidireccionales), que mediante diversas estrategias establecidas se le presentarán al alumno.

Al inicio de la aplicación, existe un proceso de identificación de las características del entorno (alumno+hardware+medios), de esta forma, la aplicación multimedia se adaptará y trabajará de acuerdo a las condiciones del entorno, donde también estará incluida una evaluación diagnóstica del entorno, contemplándose las características cognitivas del usuario y sus conocimientos previos, cabe aclarar que dicho proceso de evaluación se encuadra "como un proceso sistemático de recogida de información válida y fiable para formar juicios de valor sobre elementos y hechos educativos, de acuerdo con unos criterios, con el fin de valorarlos y tomar decisiones" (Lopez Cubino, 1997, p. 19). En consecuencia, lo que se persigue con el proceso de evaluación es recoger los datos necesarios para actuar en consecuencia.

Estos softwares, podrán tener la capacidad de poder acceder a módulos pertenecientes a otros sistemas utilizando una red, de esta forma podrá acceder a otro banco de módulos pertenecientes a otros alumnos y tener la alternativa de comunicarse con ellos para poder proporcionar el aprendizaje colaborativo, este sistema en red viene a componer otra complejidad de mayor nivel a la que se ha descrito anteriormente, formando un Sistema Macro de Aprendizaje Multimedia Interactivo.

\section{CONCLUSIONES}

La inserción del pensamiento complejo nos lleva a adentrarnos en un mundo complejo donde el determinismo da lugar al error y a la incertidumbre. Por lo tanto, la programación de aplicaciones multimedia Revista Iberoamericana de Educación (ISSN: 1681-5653) 
deben girar hacia el mismo sentido, ya que no vale de mucho la programación única y a largo plazo para diversos usuarios con perfiles tan complejos como disímiles, asimismo el trabajo a medida (mediante el almacenamiento sesgado de acuerdo a los requerimientos de cada alumno), compartido y ramificado de los SIMs, nos dará la pauta hacia donde va y que se espera conseguir con las nuevas tecnologías aplicadas a la educación.

Como afirma Morin (2001, p. 27) "un paradigma puede al mismo tiempo dilucidar y cegar, revelar y ocultar"; es así que este modelo pretende iluminar el plano complejo, lo real con lo imaginario, todavía en penumbras, que presenta el desarrollo de softwares interactivos.

Se debe dar paso a la atomización, mediante el desarrollo de parcelas de instrucciones modulares pero a la vez también al crecimiento complejo mediante la conexión y compartir de dchos módulos con secuencias en todos los sentidos, formando una gran red donde cada uno de los softwares usados por cada alumno constituye un elemento constitutivo de otro sistema macro que los contiene a todos. Toda esta red enlazadas constituirá múltiples y diversos caminos que recorra el alumno para que sea verdaderamente un trayecto individual y global a la vez.

El mantenimiento de una entropía constante tanto en el diseño de programas interactivos educativos como en su desarrollo posterior mientras se lo utiliza, nos lleva a reflexionar un nuevo tipo de materiales interactivos, incorporando elementos neguentrópicos aún no considerados, y que se hacen necesarios para un abordaje más productivo cuando interactúa con el alumno.

En la actualidad, muchos de los elementos que comprenden el modelo propuesto de SIMs no están consideradas en su totalidad por los softwares de autor, quizás sea necesario reconsiderar las herramientas de autor que existen, para adaptarlas a una nueva perspectiva más orientada a la propia construcción del conocimiento por parte del alumno, tomando el rol principal de su propio aprendizaje.

\section{BIBLIOGRAFÍA}

andrade, E., Pachano, E., Pereira, L. Torres, A. (2002). El paradigma complejo. Cinta de Moebio, 14. URL: http://www.moebio.uchile.cl/14/frames07.htm

ATKINS, P. (1991). Fisicoquímica. Wilmington: Addison-Wesley Iberoamericana.

AUSTIN MILLÁN, T. (2000). Teoría de sistemas y sociedad. URL: http://www.geocities.com/tomasaustin_cl/index.html

BRUNER, J. (1984). Acción, pensamiento y lenguaje. Madrid: Alianza.

CAstellan, G. (1987). Fisicoquímica. Wilmington: Addison-Wesley Iberoamericana.

CATAlDI, Z., Lage, F., Pessacq, R. y García Martínez, R. (2003). Revisión de marcos teóricos educativos para el diseño y uso de programas didácticos.

CAZAU, P. (1995). La teoría del caos. URL: http://galeon.com/pcazau/artfis_caos.htm

GARDNER, H. (2003). Inteligencias múltiples. Buenos Aires: Paidos.

GIMENO SACRISTÁN, J. y PÉREZ GÓMEZ, A. (1995). Comprender y Transformar la Enseñanza. Madrid: Morata.

GLASSTONE, S. (1972). Tratado de química física. Madrid: Aguilar. 
Grün, E. (1999). Una visión sistémica y cibernética del derecho. URL: http://www.filosofiayderecho.com/rtfd/numero3/grun.htm

LÓPEZ CUBINO, R. (1998). La evaluación en el área de tecnología. Salamanca: Amarú.

MARTON, P. (2003). La concepción pedagógica de sistemas de aprendizaje multimedia interactivo: fundamentos, metodología y problemática. URL: http://www. fse.ulaval.ca/graim/facteru-esp.htm

MORIN, E. (1999). La cabeza bien puesta. Buenos Aires: Nueva Visión.

MORIN, E. (2001). Los siete saberes necesarios para la educación del futuro. Buenos Aires: Nueva Visión.

PIAGET, J. (1981). Psicología y pedagogía. Barcelona: Ariel.

SÁNCHEZ CEREZO, S. (1991). Léxicos tecnología de la educación. Madrid: Santillana.

SARABIA, A. (1995). La teoría general de sistemas. Madrid: Isdefe.

SQUIRES, D. (1999). Educational software and learning: subversive use and volatile design. IEEE Proceedings of the $32^{\text {nd }}$ International Conference on System Sciences, Hawaii. URL: http://www.computer.org/proceedings/hicss/0001/00011/00011079.PDF

VAN DAM, A. (2002). Next generation educational software and next generation user interfaces. Grand Challenges

URL: http://www.cra.org/Activities/grand.challenges/vandam.pdf. 